The Astrophysical Journal, 651:775-781, 2006 November 10

(C) 2006. The American Astronomical Society. All rights reserved. Printed in U.S.A.

\title{
A REVERBERATION-BASED MASS FOR THE CENTRAL BLACK HOLE IN NGC 4151
}

\author{
Misty C. Bentz, ${ }^{1}$ Kelly D. Denney, ${ }^{1}$ Edward M. Cackett, ${ }^{2}$ Matthias Dietrich, ${ }^{1}$ Jeffrey K. J. Fogel, ${ }^{3}$ Himel Ghosh, ${ }^{1}$ \\ Keith Horne, ${ }^{2}$ Charles Kuehn, ${ }^{1,4}$ Takeo Minezaki, ${ }^{5}$ Christopher A. Onken, ${ }^{1,6}$ Bradley M. Peterson, ${ }^{1}$ \\ Richard W. Pogge, ${ }^{1}$ Vladimir I. Pronik, ${ }^{7,8}$ Douglas O. Richstone, ${ }^{3}$ Sergey G. Sergeev, ${ }^{7,8}$ \\ Marianne Vestergaard, ${ }^{9}$ Matthew G. Walker, ${ }^{3}$ and Yuzuru Yoshit ${ }^{5,10}$ \\ Received 2006 May 2; accepted 2006 June 30
}

\begin{abstract}
We have undertaken a new ground-based monitoring campaign to improve the estimates of the mass of the central black hole in NGC 4151. We measure the lag time of the broad $\mathrm{H} \beta$ line response compared to the optical continuum at $5100 \AA$ and find a lag of $6.6_{-0.8}^{+1.1}$ days. We combine our data with the recent reanalysis of UV emission lines by Metzroth and coworkers to calculate a weighted mean of the black hole mass, $M_{\mathrm{BH}}=\left(4.57_{-0.47}^{+0.57}\right) \times 10^{7} M_{\odot}$. The absolute calibration of the black hole mass is based on normalization of the AGN black hole mass-stellar velocity dispersion $\left(M_{\mathrm{BH}^{-}} \sigma_{*}\right)$ relationship to that of quiescent galaxies by Onken and coworkers. The scatter in the $M_{\mathrm{BH}}-\sigma_{*}$ relationship suggests that reverberation-mapping-based mass measurements are typically uncertain by a factor of 3-4.
\end{abstract}

Subject headings: galaxies: active — galaxies: nuclei — galaxies: Seyfert

\section{INTRODUCTION}

Reverberation mapping (Blandford \& McKee 1982; Peterson 1993 ) is the process of measuring the time lag of variations in the emission lines of an active galactic nucleus (AGN) relative to variations in the continuum source due to light travel time effects. The time lag and the width of the emission line is directly related to the mass of the central black hole $\left(M_{\mathrm{BH}}\right)$. Reverberationmapping efforts over the past 15 years have led to the compilation of broad-line region (BLR) radius measurements and black hole mass estimates for 36 active galaxies (Peterson et al. 2004, 2005). Peterson et al. (2004) undertook a reanalysis of most of the reverberation-mapping data. The data were reanalyzed in a consistent way, paying particular attention to recent improvements in cross-correlation analysis, measuring the line widths in the variable part of the emission line, and accounting for the fact that changes in the mean luminosity of the source could cause emission-line time lags to vary over longer timescales than the reverberation timescale (Peterson et al. 2002). While the end

\footnotetext{
1 Department of Astronomy, The Ohio State University, 140 West 18th Avenue, Columbus, OH 43210; bentz@astronomy.ohio-state.edu, denney@astronomy .ohio-state.edu, dietrich@astronomy.ohio-state.edu, ghosh@astronomy.ohio-state .edu,peterson@astronomy.ohio-state.edu,pogge@astronomy.ohio-state.edu.

2 School of Physics and Astronomy, University of St. Andrews, Fife KY16 9SS, Scotland, UK; emc14@st-and.ac.uk, kdh1@st-and.ac.uk.

3 Department of Astronomy, University of Michigan, Ann Arbor, MI 481091090; fogel@umich.edu, dor@umich.edu,mgwalker@umich.edu.

4 Current address: Physics and Astronomy Department, 3270 Biomedical Physical Sciences Building, Michigan State University, East Lansing, MI 48824; kuehncha@msu.edu.

5 Institute of Astronomy, School of Science, University of Tokyo, 2-21-1 Osawa, Mitaka, Tokyo 181-0015, Japan; minezaki@mtk.ioa.s.u-tokyo.ac.jp, yoshii@mtk ioa.s.u-tokyo.ac.jp.

${ }^{6}$ Current address: National Research Council Canada, Herzberg Institute of Astrophysics, 5071 West Saanich Road, Victoria, BC V9E 2E7, Canada; christopher .onken@nrc-cnrc.gc.ca.

7 Crimean Astrophysical Observatory, p/o Nauchny, 98409 Crimea, Ukraine; sergeev@crao.crimea.ua, vpronik@crao.crimea.ua.

8 Isaak Newton Institute of Chile, Crimean Branch, Ukraine.

9 Steward Observatory, University of Arizona, 933 North Cherry Avenue, Tucson, AZ 85721; mvestergaard@as.arizona.edu.

${ }_{10}$ Research Center for the Early Universe, School of Science, University of Tokyo, 7-3-1 Hongo, Bunkyo-ku, Tokyo 113-0033, Japan.
}

result was to significantly decrease the amount of random and systematic errors in a very inhomogeneous database, there remained a few data sets for individual objects that were problematic in some way, making it difficult to determine either time lags and/ or line width measurements with high confidence. NGC 4151, one of the brightest and best-studied AGNs, is one such source. Previous monitoring studies of NGC 4151 include those by Maoz et al. (1991) and Kaspi et al. (1996).

Our motivation for undertaking a new reverberation-mapping campaign on NGC 4151 is twofold. First, reverberation mapping is still the only direct method for estimating central masses in a broad population of active galaxies and is more valuable still in that it does not depend on angular resolution. Reverberation results also provide the fundamental calibration for mass estimates based on radius-luminosity scaling relationships (Wandel et al. 1999; Vestergaard 2002, 2004; McLure \& Jarvis 2002; Kollmeier et al. 2006; Vestergaard \& Peterson 2006). Second, NGC 4151 is one of the best candidates for measuring the black hole mass by other means and therefore affording a direct comparison between reverberation-based masses and other methods. Our specific goal in undertaking this new ground-based monitoring program is to significantly improve measurement of the lag, or mean response time, between the continuum and the $\mathrm{H} \beta$ emission line, the width of the $\mathrm{H} \beta$ line in the variable part of the spectrum, and the black hole mass for NGC 4151.

\section{OBSERVATIONS AND DATA REDUCTION}

\subsection{Spectroscopy}

On every clear night between 2005 February 27 and 2005 April 10, we obtained spectra of the nucleus of NGC 4151-a weakly barred Sab Seyfert galaxy at $z=0.00332$-with the Boller and Chivens CCD Spectrograph (CCDS) on the MDM Observatory $1.3 \mathrm{~m}$ McGraw-Hill Telescope. We used a $5^{\prime \prime}$ slit with a position angle (P.A.) of $90^{\circ}$, and the typical exposure time was $1200 \mathrm{~s}$. The spectra were reduced in the usual way using IRAF ${ }^{11}$

${ }^{11}$ IRAF is distributed by the National Optical Astronomical Observatory, which is operated by the Association of Universities for Research in Astronomy, Inc., under cooperative agreement with the NSF. 
and XVista. ${ }^{12}$ An extraction width of 17 pixels, corresponding to 12 .'75, was used in the spectral reduction. Each night had between two and four individual spectra of NGC 4151, which were averaged together to increase the signal-to-noise ratio.

Additional spectra were obtained with the Nasmith Spectrograph and Astro-550 $580 \times 520$ pixel CCD (Berezin et al. 1991) at the Crimean Astrophysical Observatory (CrAO) $2.6 \mathrm{~m}$ Shajn Telescope. Spectra were obtained through a $3^{\prime \prime}$ slit at P.A. $=90^{\circ}$ and were reduced in the usual way. An extraction width of 16 pixels, corresponding to $11^{\prime \prime}$, was used in the reduction.

Once the spectra were reduced, they were internally calibrated within each data set. Because the [O III] narrow lines are very bright in NGC 4151, we had saturation problems with the $\lambda 5007$ line, and therefore scaled all the spectra using the $\lambda 4959$ line. This was accomplished by employing the spectral scaling software of van Groningen \& Wanders (1992). A mean spectrum derived from all of the data in each set was used as the reference spectrum. The individual spectra were compared to the reference spectra and scaled so as to minimize the residuals of the [O III $\lambda 4959$ line in the difference spectrum (produced by subtracting the reference spectrum from the individual spectrum). This process resulted in a common [O III] $\lambda 4959$ flux of $3.76 \times 10^{-12} \mathrm{ergs} \mathrm{s}^{-1}$ $\mathrm{cm}^{-2}$. As the flux ratio of the [O III] $\lambda 5007$ to the [O III $\lambda 4959$ line is 2.94, our measurement of the [O III] $\lambda 4959$ flux is consistent with the values of $F([\mathrm{O} \mathrm{III}])=1.575 \times 10^{-11} \mathrm{ergs} \mathrm{s}^{-1} \mathrm{~cm}^{-2}$ for the combined [O III] flux determined by Kaspi et al. (1996) and $F\left(\left[\mathrm{O}_{\mathrm{III}}\right] 25007\right)=1.19 \pm 0.06 \times 10^{-11} \mathrm{ergs} \mathrm{s}^{-1} \mathrm{~cm}^{-2}$ determined by Antonucci \& Cohen (1983).

The light curves were created by measuring the average continuum flux between the observed-frame wavelengths of 5100 and $5130 \AA$. To measure the $\mathrm{H} \beta$ line flux, we use observed-frame wavelength windows of $4770-4800$ and $5100-5130 \AA$ to estimate a linear continuum and then integrate the flux above the best-fit continuum between 4810 and $4940 \AA$.

\subsection{Photometry}

Photometric $V$-band data were obtained with the multicolor imaging photometer (MIP) at the $2.0 \mathrm{~m}$ Multicolor Active Galactic Nuclei Monitoring (MAGNUM) Telescope at the Haleakala Observatories in Hawaii (Kobayashi et al. 1998a, 1998b; Yoshii 2002; Yoshii et al. 2003). The telescope was pointed first at NGC 4151 and then at two reference stars with $(\Delta \alpha, \Delta \delta)=$ $\left(16 !^{\prime} 1,5 ! 7\right)$ and $(-11.3,-9.3)$, after which the telescope was dithered. The reference stars were previously determined to be nonvariable (Minezaki et al. 2004).

The images were reduced with IRAF in the usual way, with an additional small correction applied for the nonlinearity of the detector. Aperture photometry with an aperture diameter of 8.'3 was applied to the nucleus of NGC 4151 and the two reference stars, with sky flux measured between radii of 5.5 and 6." 9 . The nuclear flux of NGC 4151 relative to the reference star at $(\Delta \alpha, \Delta \delta)=$ $\left(16{ }^{\prime} 1,5.7\right)$ was estimated to be the average of the relative fluxes for all of the dithering sets. The reference star flux was calibrated with respect to photometric standard stars taken from Landolt (1992) and Hunt et al. (1998).

\subsection{Intercalibration of Light Curves}

Each of these data sets involve different aperture geometries and seeing conditions, which result in different slit losses and

\footnotetext{
12 XVISTA was originally developed as Lick Observatory Vista and is now maintained in the public domain by former Lick graduate students as a service to the community. It is currently maintained by Jon Holtzman at New Mexico State University and is available at http://ganymede.nmsu.edu/holtz/xvista.
}

varying amounts of starlight contamination. Therefore, they must be intercalibrated to fit each other before merging them into a final light curve. The MDM data set was the largest and was therefore the anchor to which the CrAO and MAGNUM data were scaled. Following the discussion of Peterson et al. (1991), the CrAO and MAGNUM light curves were each compared to the MDM light curves to identify pairs of observations that were within 1 day of each other. A least-squares analysis was then performed on the sets of pairs of observations to identify the overall offsets, the results of the various monitoring geometries, between the MDM data set and the CrAO and MAGNUM data sets. After the removal of the offsets, the CrAO and MAGNUM light curves were merged with the MDM light curves.

The full light curves are given in Table 1. For our analysis, we binned all observations that occurred within a 0.5 day window. The statistical properties of the binned light curves are given in Table 2. Column (1) gives the spectral feature, and column (2) gives the number of measurements in the light curve. Columns (3) and (4) are the sampling intervals between data points, measured as the mean and median, respectively. Column (5) is the mean flux and standard deviation of the light curve, not including a correction for host galaxy starlight, which, following the methods of Bentz et al. (2006) for this slit geometry, is measured to be $F_{\text {gal }}(5100 \AA)=(2.11 \pm 0.18) \times 10^{-14} \mathrm{ergs} \mathrm{s}^{-1} \mathrm{~cm}^{-2} \AA^{-1}$. Column (6) gives the mean fractional error, which is based on the comparison of observations that are closely spaced in time. The excess variance in column (7) is computed as

$$
F_{\mathrm{var}}=\frac{\sqrt{\sigma^{2}-\delta^{2}}}{\langle f\rangle}
$$

where $\sigma^{2}$ is the variance of the fluxes, $\delta^{2}$ is their mean-square uncertainty, and $\langle f\rangle$ is the mean of the observed fluxes. Finally, column (8) is the ratio of the maximum to the minimum flux for each light curve.

\section{DATA ANALYSIS}

\subsection{Time Series Analysis}

To measure the lag between the continuum and the variable part of the $\mathrm{H} \beta$ line, we cross-correlate the $\mathrm{H} \beta$ emission-line light curve with the continuum light curve measured at $5100 \AA$. We use the interpolation correlation function (ICF) method as described by White \& Peterson (1994). The light curves and cross-correlation functions are shown in Figure 1.

To better quantify the uncertainties in the time delay measurement, we employ the model-independent Monte Carlo FR/RSS method, as described by Peterson et al. (1998), including modifications described by Peterson et al. (2004). For each single realization of the method, "random subset sampling" (RSS) is employed in which a light curve with $N$ data points is randomly sampled $N$ times without regard to the previous selection of each point. A data point that is selected $M$ times will have its uncertainty reduced by a factor $M^{1 / 2}$. "Flux randomization" (FR), in which a random Gaussian deviation, based on the associated error bar, is then applied to each of the selected $N$ points. This FR/RSS-altered subset of data points is then cross-correlated as though it were real data. The peak of the cross-correlation function, $r_{\max }$, which occurs at a time lag $\tau_{\mathrm{pk}}$, is determined, as is the centroid, $\tau_{\text {cen }}$, which is computed from those points near the peak with $r \geq 0.8 r_{\max }$. A cross-correlation peak distribution (CCPD) for $\tau_{\mathrm{pk}}$ and a cross-correlation centroid distribution (CCCD) for $\tau_{\text {cen }}$ are built with a large number $(N=10,000)$ 
TABLE 1

Continuum and H $\beta$ FluXes for NGC 4151

\begin{tabular}{|c|c|c|c|}
\hline $\begin{array}{l}\text { Julian Date }^{\mathrm{a}} \\
(-2,450,000)\end{array}$ & $\begin{array}{c}F_{\lambda}(5100 \AA) \\
\left(10^{-15} \operatorname{ergs~s}^{-1} \mathrm{~cm}^{-2} \AA^{-1}\right)\end{array}$ & $\begin{array}{c}\mathrm{H} \beta \lambda 4681 \\
\left(10^{-13} \mathrm{ergs} \mathrm{s}^{-1} \mathrm{~cm}^{-2}\right)\end{array}$ & Observatory Code ${ }^{b}$ \\
\hline $3430.020 \ldots \ldots \ldots \ldots \ldots \ldots \ldots$ & $26.96 \pm 0.62$ & $21.40 \pm 0.27$ & M \\
\hline 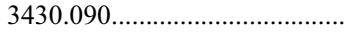 & $26.12 \pm 0.43$ & $\ldots$ & $\mathrm{H}$ \\
\hline $3430.785 \ldots \ldots \ldots \ldots \ldots \ldots \ldots \ldots \ldots \ldots \ldots$ & $26.03 \pm 0.60$ & $21.13 \pm 0.27$ & M \\
\hline $3431.762 \ldots \ldots \ldots$ & $27.13 \pm 0.63$ & $21.90 \pm 0.28$ & M \\
\hline 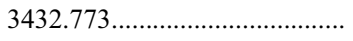 & $28.24 \pm 0.65$ & $22.42 \pm 0.28$ & M \\
\hline $3432.809 \ldots \ldots \ldots \ldots$ & $28.10 \pm 0.54$ & $\ldots$ & $\mathrm{H}$ \\
\hline $3433.750 \ldots \ldots \ldots \ldots \ldots$ & $27.18 \pm 0.63$ & $21.94 \pm 0.28$ & M \\
\hline $3434.941 \ldots \ldots \ldots \ldots \ldots \ldots \ldots \ldots$ & $28.55 \pm 0.63$ & $\ldots$ & $\mathrm{H}$ \\
\hline $3436.910 \ldots \ldots \ldots \ldots \ldots \ldots \ldots \ldots \ldots \ldots \ldots$ & $29.87 \pm 0.46$ & $\ldots$ & $\mathrm{H}$ \\
\hline $3437.336 \ldots \ldots \ldots \ldots \ldots \ldots \ldots \ldots \ldots \ldots \ldots \ldots$ & $31.62 \pm 0.70$ & $22.41 \pm 0.40$ & $\mathrm{C}$ \\
\hline 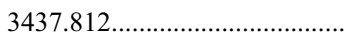 & $28.14 \pm 0.65$ & $23.33 \pm 0.29$ & M \\
\hline 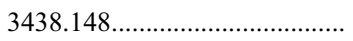 & $29.00 \pm 0.27$ & $\ldots$ & $\mathrm{H}$ \\
\hline $3438.750 \ldots \ldots \ldots \ldots \ldots \ldots \ldots$ & $28.35 \pm 0.66$ & $23.69 \pm 0.30$ & M \\
\hline $3439.723 \ldots \ldots \ldots \ldots \ldots \ldots \ldots \ldots \ldots$ & $27.10 \pm 0.63$ & $23.37 \pm 0.29$ & M \\
\hline $3440.754 \ldots \ldots \ldots \ldots \ldots \ldots \ldots \ldots \ldots \ldots \ldots \ldots$ & $26.03 \pm 0.60$ & $23.28 \pm 0.29$ & M \\
\hline 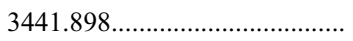 & $24.89 \pm 0.58$ & $23.88 \pm 0.30$ & M \\
\hline $3442.754 \ldots \ldots \ldots \ldots \ldots \ldots \ldots \ldots$ & $23.53 \pm 0.54$ & $23.38 \pm 0.30$ & M \\
\hline $3442.820 \ldots \ldots \ldots \ldots \ldots \ldots \ldots \ldots \ldots \ldots \ldots \ldots \ldots$ & $24.15 \pm 1.15$ & $\ldots$ & $\mathrm{H}$ \\
\hline 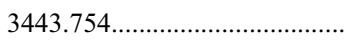 & $22.38 \pm 0.52$ & $23.24 \pm 0.29$ & M \\
\hline 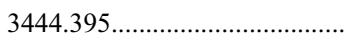 & $22.90 \pm 0.51$ & $23.08 \pm 0.42$ & $\mathrm{C}$ \\
\hline 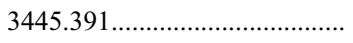 & $21.43 \pm 0.47$ & $22.69 \pm 0.41$ & $\mathrm{C}$ \\
\hline $3445.766 \ldots \ldots \ldots \ldots$ & $21.49 \pm 0.50$ & $22.36 \pm 0.28$ & M \\
\hline $3446.332 \ldots \ldots \ldots \ldots \ldots \ldots \ldots$ & $21.28 \pm 0.47$ & $21.56 \pm 0.39$ & $\mathrm{C}$ \\
\hline $3446.746 \ldots \ldots \ldots \ldots \ldots \ldots \ldots \ldots$ & $21.96 \pm 0.51$ & $21.85 \pm 0.28$ & M \\
\hline 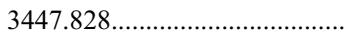 & $21.09 \pm 0.69$ & $\ldots$ & $\mathrm{H}$ \\
\hline $3447.930 \ldots \ldots \ldots \ldots \ldots \ldots \ldots \ldots \ldots \ldots$ & $21.66 \pm 0.50$ & $20.83 \pm 0.26$ & M \\
\hline $3450.785 \ldots \ldots \ldots \ldots \ldots \ldots \ldots \ldots \ldots$ & $21.64 \pm 0.50$ & $19.76 \pm 0.25$ & M \\
\hline $3451.777 \ldots \ldots \ldots$ & $21.62 \pm 0.50$ & $19.34 \pm 0.24$ & M \\
\hline $3451.988 \ldots \ldots \ldots \ldots$ & $22.14 \pm 0.16$ & $\ldots$ & $\mathrm{H}$ \\
\hline $3452.695 \ldots \ldots \ldots \ldots \ldots \ldots \ldots \ldots . .$. & $21.65 \pm 0.50$ & $18.99 \pm 0.24$ & M \\
\hline $3456.766 \ldots \ldots \ldots \ldots \ldots \ldots \ldots \ldots \ldots$ & $22.07 \pm 0.51$ & $19.01 \pm 0.24$ & M \\
\hline $3458.910 \ldots \ldots \ldots \ldots \ldots \ldots \ldots \ldots \ldots \ldots \ldots \ldots$ & $21.90 \pm 0.51$ & $17.89 \pm 0.23$ & M \\
\hline 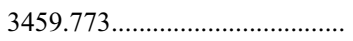 & $21.25 \pm 0.49$ & $18.10 \pm 0.23$ & M \\
\hline $3460.863 \ldots \ldots \ldots \ldots \ldots \ldots \ldots \ldots \ldots$ & $21.74 \pm 0.50$ & $18.00 \pm 0.23$ & M \\
\hline $3461.746 \ldots \ldots \ldots \ldots$ & $21.67 \pm 0.50$ & $17.86 \pm 0.23$ & M \\
\hline $3462.781 \ldots \ldots \ldots \ldots \ldots \ldots \ldots \ldots$ & $21.29 \pm 0.49$ & $17.61 \pm 0.22$ & M \\
\hline 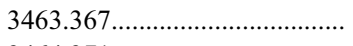 & $23.37 \pm 0.52$ & $17.74 \pm 0.32$ & $\mathrm{C}$ \\
\hline 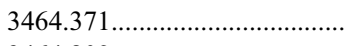 & $23.02 \pm 0.51$ & $17.52 \pm 0.32$ & $\mathrm{C}$ \\
\hline $3464.809 \ldots \ldots \ldots \ldots \ldots \ldots \ldots \ldots \ldots \ldots \ldots$ & $22.27 \pm 0.52$ & $18.00 \pm 0.23$ & M \\
\hline 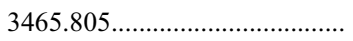 & $21.93 \pm 0.51$ & $17.52 \pm 0.22$ & M \\
\hline $3466.793 \ldots \ldots \ldots$ & $22.68 \pm 0.52$ & $17.29 \pm 0.22$ & M \\
\hline 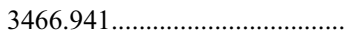 & $21.87 \pm 0.16$ & $\ldots$ & $\mathrm{H}$ \\
\hline 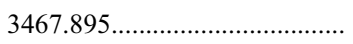 & $22.03 \pm 0.51$ & $17.55 \pm 0.22$ & M \\
\hline $3468.781 \ldots \ldots \ldots \ldots \ldots \ldots \ldots \ldots \ldots \ldots$ & $20.66 \pm 0.48$ & $17.70 \pm 0.22$ & M \\
\hline 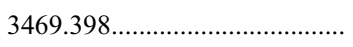 & $21.01 \pm 0.46$ & $17.46 \pm 0.31$ & $\mathrm{C}$ \\
\hline $3469.734 \ldots \ldots \ldots \ldots \ldots \ldots \ldots \ldots . .$. & $20.80 \pm 0.48$ & $17.56 \pm 0.22$ & M \\
\hline $3469.941 \ldots \ldots \ldots \ldots$ & $20.64 \pm 0.11$ & $\ldots$ & $\mathrm{H}$ \\
\hline $3470.312 \ldots \ldots \ldots \ldots \ldots \ldots \ldots \ldots \ldots \ldots$ & $20.80 \pm 0.46$ & $17.28 \pm 0.31$ & $\mathrm{C}$ \\
\hline $3470.785 \ldots \ldots \ldots \ldots \ldots \ldots \ldots$ & $20.30 \pm 0.47$ & $17.46 \pm 0.22$ & M \\
\hline $3471.785 \ldots \ldots \ldots \ldots$ & $20.66 \pm 0.48$ & $16.90 \pm 0.21$ & M \\
\hline
\end{tabular}

\footnotetext{
a The Julian Date listed is the midpoint of the observation.

${ }^{\mathrm{b}}$ Observatory codes: (C) CrAO $2.6 \mathrm{~m}$ Shajn Telescope + Nasmith Spectrograph; (H) Haleakala Observatories $2.0 \mathrm{~m}$ MAGNUM Telescope + MIP; (M) MDM Observatory $1.3 \mathrm{~m}$ McGraw-Hill Telescope + CCDS.
}

of Monte Carlo realizations. We take the average value of the CCPD to be $\tau_{\mathrm{pk}}$ and the average value of the CCCD to be $\tau_{\mathrm{cen}}$, with uncertainties $\Delta \tau_{\text {up }}$ and $\Delta \tau_{\text {low }}$ such that $15.87 \%$ of the CCCD realizations have values $\tau>\tau_{\text {cen }}+\Delta \tau_{\text {up }}$ and $15.87 \%$ have values $\tau<\tau_{\text {cen }}-\Delta \tau_{\text {low }}$, with similar uncertainties for $\tau_{\text {pk }}$. These definitions of the uncertainty correspond to $\pm 1 \sigma$ for a Gaussian distribution. We find an observed-frame lag time of $\tau_{\text {cen }}=$ $6.59_{-0.76}^{+1.12}$ days and $\tau_{\mathrm{pk}}=6.10_{-0.60}^{+1.20}$ days for NGC 4151 .

\subsection{Line Width Measurement}

In order to calculate the black hole mass, we need to measure the width of the $\mathrm{H} \beta$ line in the variable (rms) part of the spectrum. The mean and rms spectra, shown in Figure 2, were calculated using the full set of MDM spectra. To measure the line width, we first interpolate the rms continuum underneath the $\mathrm{H} \beta$ emission line by choosing continuum windows on either side of the 
TABLE 2

Light-Curve Statistics

\begin{tabular}{|c|c|c|c|c|c|c|c|}
\hline $\begin{array}{l}\text { Time Series } \\
\text { (1) }\end{array}$ & $\begin{array}{l}N \\
(2)\end{array}$ & $\begin{array}{c}\langle T\rangle \\
\text { (days) } \\
(3)\end{array}$ & $\begin{array}{c}T_{\text {median }} \\
\text { (days) } \\
(4)\end{array}$ & $\begin{array}{c}\text { Mean Flux } \\
\text { (5) }\end{array}$ & $\begin{array}{l}\text { Mean Fractional Error } \\
\text { (6) }\end{array}$ & $\begin{array}{l}F_{\text {var }} \\
(7)\end{array}$ & $\begin{array}{l}R_{\max } \\
(8)\end{array}$ \\
\hline $5100 \AA .$. & 37 & 1.2 & 1.0 & $23.8 \pm 3.0$ & 0.019 & 0.124 & $1.45 \pm 0.03$ \\
\hline $\mathrm{H} \beta \ldots \ldots \ldots \ldots \ldots \ldots$ & 34 & 1.3 & 1.0 & $20.1 \pm 2.5$ & 0.012 & 0.121 & $1.41 \pm 0.02$ \\
\hline
\end{tabular}

${ }^{a}$ Fluxes are in $10^{-15} \mathrm{ergs} \mathrm{s}^{-1} \mathrm{~cm}^{-2} \AA^{-1}$ for $5100 \AA$ and $10^{-13} \mathrm{ergs} \mathrm{s}^{-1} \mathrm{~cm}^{-2}$ for $\mathrm{H} \beta$.

line. The line width is then characterized by its full width at half-maximum (FWHM) and by the line dispersion (the second moment of the line profile) $\sigma_{\text {lin }}$, as described by Peterson et al. (2004). In order to characterize the uncertainties in each of these measurements, we use the following procedure, as described by Peterson et al. (2004). For a set of $N$ spectra, we select $N$ spectra at random, without regard to whether a spectrum has been previously selected or not. The $N$ randomly selected spectra are then used to construct mean and rms spectra from which the line width measurements are made. This process is one Monte Carlo realization, and a large number $(N=10,000)$ of these realizations gives a mean and standard deviation for each of the measurements of the line width. The $\mathrm{H} \beta$ emission-line width measurements we determined from the rms spectrum for NGC 4151 are $\sigma_{\operatorname{lin}}=$ $2680 \pm 64 \mathrm{~km} \mathrm{~s}^{-1}$ and FWHM $=4711 \pm 750 \mathrm{~km} \mathrm{~s}^{-1}$ in the rest frame of NGC 4151 .

\subsection{Black Hole Mass}

The mass of the black hole is given by

$$
M_{\mathrm{BH}}=\frac{f_{c} \tau \Delta V^{2}}{G},
$$

where $\tau$ is the emission-line time delay, $\Delta V$ is the width of the emission line, and $G$ is the gravitational constant. The factor $f$

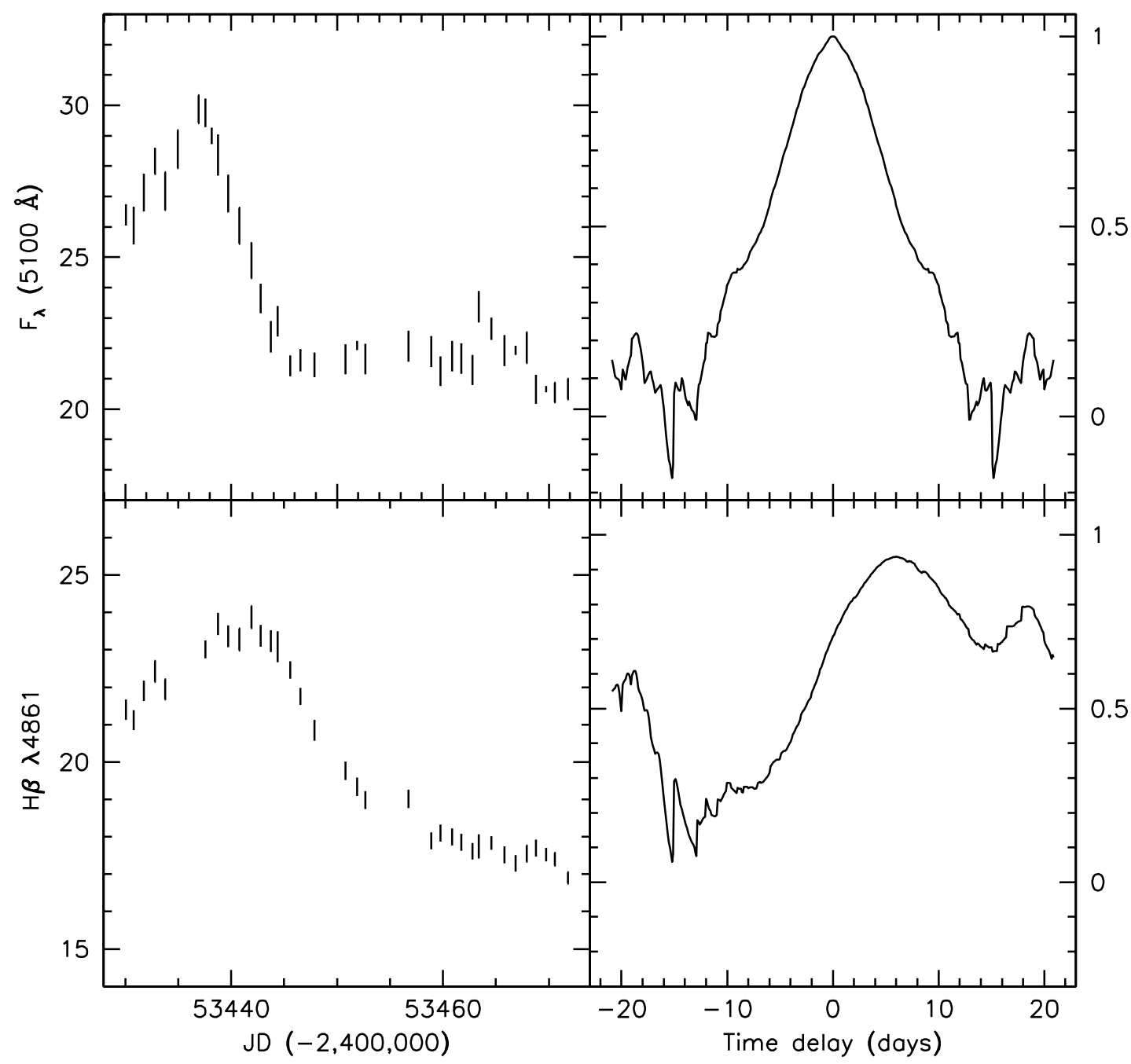

FIG. 1. L Left panels: Light curves for the continuum region at $5100 \AA \AA$ and for the $\mathrm{H} \beta \lambda 4861$ line. Measurements taken within a 0.5 day bin have been averaged together. The continuum flux is in units of $10^{-15} \mathrm{ergs} \mathrm{s}^{-1} \mathrm{~cm}^{-2} \AA^{-1}$, and the $\mathrm{H} \beta$ flux is in units of $10^{-13} \mathrm{ergs} \mathrm{s}^{-1} \mathrm{~cm}^{-2}$. Right panels: Results of cross-correlation with the continuum light curve. The top right panel is, therefore, the continuum autocorrelation function. 


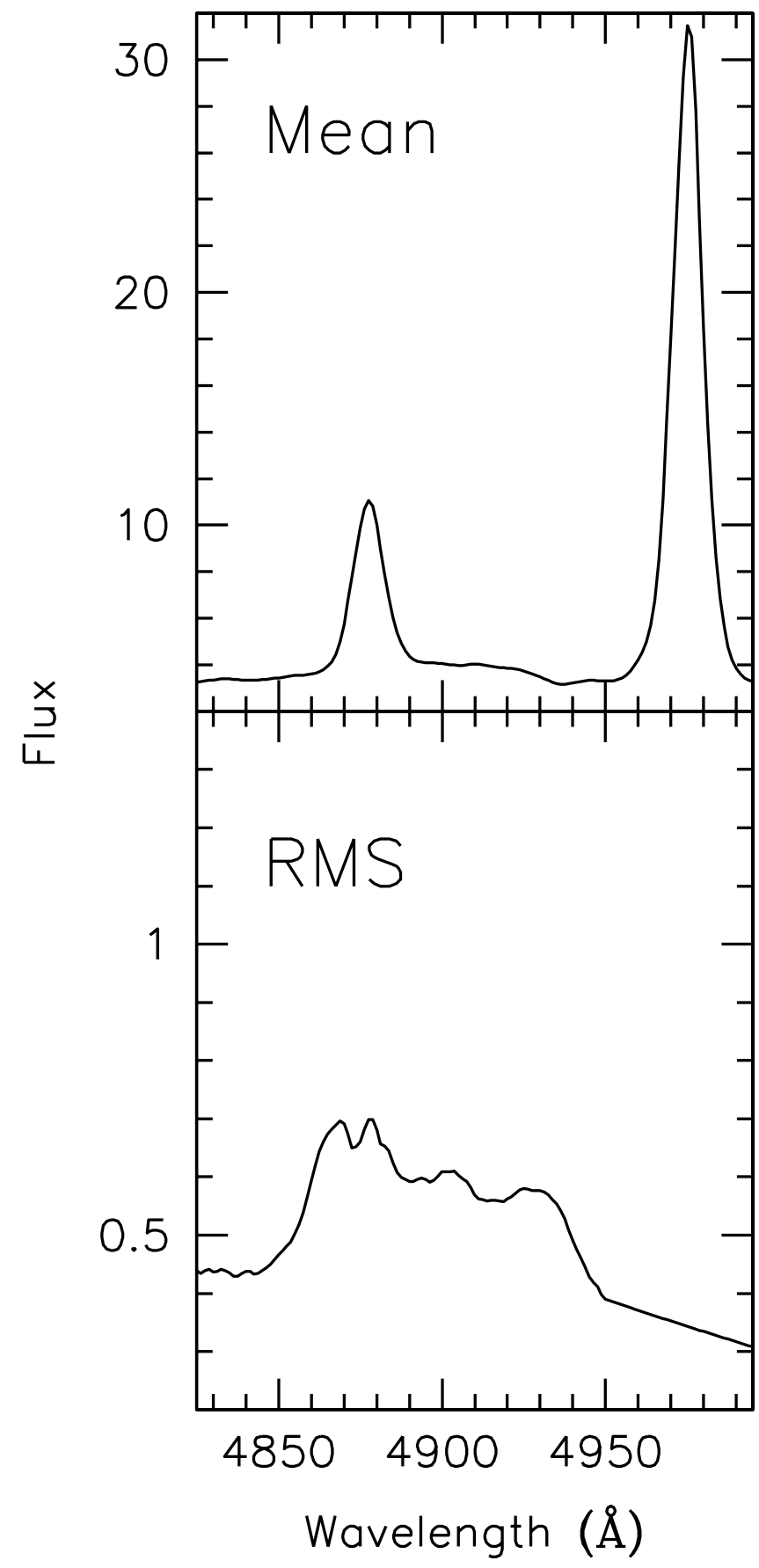

FIG. 2.-Mean and rms of the MDM spectra in the observed frame of NGC 4151. The narrow components of the lines have been subtracted from each spectrum before creating the rms spectrum to avoid the residuals of the very strong [O III] lines in NGC 4151. Some of the structure along the top of the $\mathrm{H} \beta$ broad line is likely due to the imperfect subtraction of the narrow component of the line. The flux for both spectra is in units of $10^{-14} \mathrm{ergs} \mathrm{s}^{-1} \mathrm{~cm}^{-2} \AA^{-1}$.

depends on the geometry, kinematics, and inclination of the BLR and has been shown by Onken et al. (2004) to have an average value of $\langle f\rangle=5.5$ when normalizing the AGN relationship between black hole mass and stellar velocity dispersion $\left(M_{\mathrm{BH}^{-}} \sigma_{*}\right)$ to that of quiescent galaxies.

To estimate the mass of NGC 4151, we use $\tau_{\text {cen }}$ for the time delay and $\sigma_{\text {lin }}$ for the line width of $\mathrm{H} \beta$. We find $M_{\mathrm{BH}}=\left(5.1_{-0.6}^{+0.9}\right) \times$ $10^{7} M_{\odot}$. We would, however, like to remind the reader that all reverberation-based masses are likely to be uncertain by a factor of 3-4.

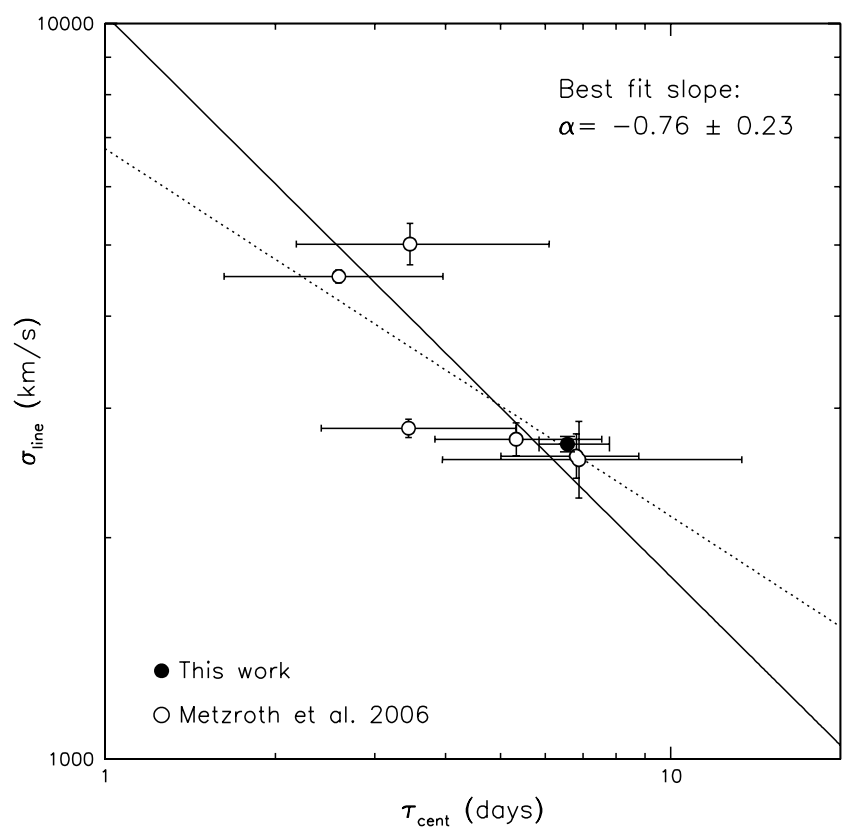

FIG. 3.- Rest-frame emission-line widths vs. time lags for lines in NGC 4151. The filled circle is the data from this work. The open circles are UV emission lines from Metzroth et al. (2006). The solid line has a slope of $-0.76 \pm 0.24$ and is the best fit to the circles. The dotted line has a forced slope of -0.5 , i.e., a virial relationship.

\section{DISCUSSION AND CONCLUSIONS}

It has been clear for many years that the BLRs in AGNs are stratified in ionization. Emission lines arising from regions of more highly ionized gas have shorter time lags, and lines arising from regions of less highly ionized gas have longer time lags (Clavel et al. 1991; Peterson et al. 1991; Dietrich et al. 1993; Maoz et al. 1993). In addition, it has been known that lines arising from more highly ionized gas have broader profiles than lines arising from less highly ionized gas (Osterbrock \& Shuder 1982). If gravity dominates the kinematics of the BLR gas, then a virial relationship between the time lag and the emission line width, $\Delta V \propto \tau^{-1 / 2}$, will exist. In fact, this relationship has been found in NGC 5548 (Peterson \& Wandel 1999), as well as in a handful of other objects (Peterson et al. 2000; Onken \& Peterson 2002; Kollatschny 2003).

We can investigate the presence of the expected virial relationship between time lag and line width in NGC 4151 by comparing our measurements with those of other monitoring programs for NGC 4151. A reanalysis of previous ultraviolet monitoring campaigns for NGC 4151 was recently undertaken by Metzroth et al. (2006). Even though the International Ultraviolet Explorer (IUE) data set of Clavel et al. (1990) is undersampled and the IUE data set of Ulrich \& Horne (1996) is of a fairly short duration, the estimate of $M_{\mathrm{BH}}$ determined by Metzroth et al. (2006) of $(4.14 \pm 0.73) \times 10^{7} M_{\odot}$ is in good agreement with the value we obtain above.

Figure 3 shows the lag and line-width measurements (given by $\tau_{\text {cen }}$ and $\sigma_{\text {lin }}$ ) for NGC 4151 from Metzroth et al. (2006) and from this work. We fit a power law to the UV data from Metzroth et al. (2006) and the optical data from this work. Even with the small range of values available for $\tau$ and $\Delta V$, we find that the best fit gives a slope of $\alpha=-0.76 \pm 0.24$, consistent with the slope of $\alpha=-0.5$ expected if the virial relationship between the size of the BLR and the velocity of the gas exists. We do not include measurements from two earlier ground-based monitoring campaigns on NGC 4151, one in 1988 (Maoz et al. 1991) and one in 
TABLE 3

Black Hole Mass Estimates

\begin{tabular}{|c|c|c|}
\hline Description & $\begin{array}{c}M_{\mathrm{BH}} \\
\left(10^{7} M_{\odot}\right)\end{array}$ & References \\
\hline 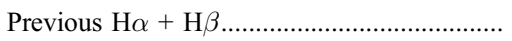 & $1.33_{-0.46}^{+0.46}$ & 1 \\
\hline UV weighted mean & $4.14_{-0.73}^{+0.73}$ & 2 \\
\hline New $\mathrm{H} \beta$ & $5.05_{-0.63}^{+0.89}$ & 3 \\
\hline $\mathrm{UV}+\mathrm{H} \beta$ weighted mean ${ }^{*} \ldots \ldots \ldots \ldots \ldots \ldots \ldots \ldots \ldots$ & $4.57_{-0.47}^{+0.57}$ & 3 \\
\hline$M_{\mathrm{BH}}{ }^{-} \sigma_{*}$ estimate & $0.62_{-0.30}^{+0.59}$ & 4,5 \\
\hline
\end{tabular}

NoтE.- The asterisk indicates the estimate determined with the $\mathrm{H} \beta$ results discussed in this work and the UV emission lines analyzed by Metzroth et al. (2006), which we take to be the best reverberation-mapping mass estimate for NGC 4151.

ReFERENCES. - (1) Peterson et al. 2004; (2) Metzroth et al. 2006; (3) this work; (4) Ferrarese et al. 2001; (5) Tremaine et al. 2002.

1993-1994 (Kaspi et al. 1996), for reasons that we briefly mention here but that are described in detail in Metzroth et al. (2006). The Kaspi et al. data set has a rather unconstrained lag time that is consistent with zero once the monotonic increase in the continuum and line flux is removed. The Maoz et al. data set, on the other hand, has a variable line spread function, as well as low spectral resolution that causes the [O III] lines to blend together. These factors make the line width determinations suspect.

Although the line widths in the Maoz et al. (1991) campaign appear to be unreliable, the time lag between the continuum and $\mathrm{H} \beta$ line is well defined. The reanalysis of this data set in Peterson et al. (2004) gives a lag time of $\tau_{\text {cen }}=11.5 \pm 3.7$ days, which is quite a bit longer than the lag time found in this study. This discrepancy is not surprising given the variable nature of such objects. For comparison, the lag time of the $\mathrm{H} \beta$ line with respect to the continuum has been observed to range between 6.5 and 26.5 days in NGC 5548 as the ionizing flux level changes over time (Peterson et al. 2004). With such a difference in the measured lag times of the $\mathrm{H} \beta$ line in NGC 4151, we would expect that the flux would be $30 \%$ higher in the Maoz et al. campaign than in the current study, $F(\mathrm{H} \beta)=(20.1 \pm 2.5) \times 10^{-13} \mathrm{ergs} \mathrm{s}^{-1}$ $\mathrm{cm}^{-2}$. In actuality, the flux during the Maoz et al. campaign, $F(\mathrm{H} \beta)=(21.5 \pm 3.4) \times 10^{-13} \mathrm{ergs} \mathrm{s}^{-1} \mathrm{~cm}^{-2}$, is $1.5 \sigma$ below the expected value. However, this is not outside the observed scatter for the $\mathrm{H} \beta$ lag times and luminosities measured for NGC 5548 (see Fig. 3 of Peterson et al. 2002).

Table 3 lists the black hole mass estimates for NGC 4151 calculated using a variety of data sets and methods. We indicated with an asterisk the weighted mean of the black hole mass estimated using the $\mathrm{H} \beta$ lag and line width and the masses determined with the UV emission lines analyzed by Metzroth et al. (2006), a value of $M_{\mathrm{BH}}=4.57_{-0.47}^{+0.57} \times 10^{7} M_{\odot}$, which we take to be the best reverberation-mapping mass estimate for NGC 4151. We can compare the mass determined using reverberation mapping to the mass expected from the $M_{\mathrm{BH}^{-}} \sigma_{*}$ relationship. The stellar velocity dispersion of NGC 4151 was measured by Ferrarese et al. (2001) to be $\sigma_{*}=93 \pm 14 \mathrm{~km} \mathrm{~s}^{-1}$. Using the slope and zero-point determined by Tremaine et al. (2002), the $M_{\mathrm{BH}}-\sigma_{*}$ relationship predicts a black hole mass of $M_{\mathrm{BH}}=6.2_{-3.0}^{+5.9} \times$ $10^{6} M_{\odot}$, with both the scatter in the $M_{\mathrm{BH}}-\sigma_{*}$ relationship and the uncertainty in the measurement of $\sigma_{*}$ accounting for the large errors in the predicted black hole mass. This is about a factor of 7 smaller than the reverberation measurement. We caution, however, that the calibration of the reverberation-based black hole mass scale (Onken et al. 2004) is averaged over many objects. The scale factor $f$ in equation (1) depends on the structure, kinematics, and inclination of the BLR (e.g., Collin et al. 2006) and is expected to be different for each AGN. Use of the mean scaling factor $\langle f\rangle=5.5$ removes bias from the sample, in the sense that as many masses are underestimated as overestimated. Given that there is a factor of 3-4 scatter around the normalized AGN $M_{\mathrm{BH}}-\sigma_{*}$ relationship, a single object deviating by a factor of 7 is hardly inconsistent.

In summary, the ground-based reverberation-mapping data presented here for NGC 4151 supersede previous optical data sets on this AGN. We have measured the lag of the $\mathrm{H} \beta$ line from the continuum flux at $5100 \AA$ and found it to be $6.6_{-0.8}^{+1.1}$ days. We have calculated the weighted mean of black hole masses determined using $\mathrm{H} \beta$, as well as the UV emission lines analyzed by Metzroth et al. (2006), which we find to be $4.57_{-0.47}^{+0.57} \times 10^{7} M_{\odot}$. We have also found that the data are consistent with a virial relationship between the size of the BLR and the velocity of BLR gas in NGC 4151.

We would like to thank an anonymous referee for comments that added to the presentation of this paper. We are grateful for support of this work by the National Science Foundation through grant AST 02-05964 to The Ohio State University and the Civilian Research and Development Foundation through grant UP 1-2549-CR-03. M. C. B. is supported by a National Science Foundation Graduate Fellowship. K. D. D. is supported by a GK-12 Fellowship of the National Science Foundation. E. M. C. gratefully acknowledges support from PPARC. M. V. acknowledges financial support from NSF grant AST 03-07384 to the University of Arizona. This research has made use of the NASA/ IPAC Extragalactic Database, which is operated by the Jet Propulsion Laboratory, California Institute of Technology, under contract with the National Aeronautics and Space Administration.
Antonucci, R. R. J., \& Cohen, R. D. 1983, ApJ, 271, 564

Bentz, M. C., Peterson, B. M., Pogge, R. W., Vestergaard, M., \& Onken, C. A. 2006, ApJ, 644, 133

Berezin, V. Y., et al. 1991, Soviet Astron. Lett., 17, 405

Blandford, R. D., \& McKee, C. F. 1982, ApJ, 255, 419

Clavel, J., et al. 1990, MNRAS, 246, 668 1991, ApJ, 366, 64

Collin, S., Kawaguchi, T., Peterson, B. M., \& Vestergaard, M. 2006, A\&A, 456, 75

Dietrich, M., et al. 1993, ApJ, 408, 416

Ferrarese, L., Pogge, R. W., Peterson, B. M., Merritt, D., Wandel, A., \& Joseph, C. L. 2001, ApJ, 555, L79

Hunt, L. K., Mannucci, F., Testi, L., Migliorini, S., Stanga, R. M., Baffa, C., Lisi, F., \& Vanzi, L. 1998, AJ, 115, 2594

Kaspi, S., et al. 1996, ApJ, 470, 336

\section{REFERENCES}

Kobayashi, Y., Yoshii, Y., Peterson, B. A., Minezaki, T., Enya, K., Suganuma, M., \& Yamamuro, T. 1998a, Proc. SPIE, 3354, 769

Kobayashi, Y., et al. 1998b, Proc. SPIE, 3352, 120

Kollatschny, W. 2003, A\&A, 407, 461

Kollmeier, J. A., et al. 2006, ApJ, 648, 128

Landolt, A. U. 1992, AJ, 104, 340

Maoz, D., et al. 1991, ApJ, 367, 493

1993, ApJ, 404, 576

McLure, R. J., \& Jarvis, M. J. 2002, MNRAS, 337, 109

Metzroth, K. G., Onken, C. A., \& Peterson, B. M. 2006, ApJ, 647, 901

Minezaki, T., Yoshii, Y., Kobayashi, Y., Enya, K., Suganuma, M., Tomita, H., Aoki, T., \& Peterson, B. A. 2004, ApJ, 600, L35

Onken, C. A., Ferrarese, L., Merritt, D., Peterson, B. M., Pogge, R. W., Vestergaard, M., \& Wandel, A. 2004, ApJ, 615, 645

Onken, C. A., \& Peterson, B. M. 2002, ApJ, 572, 746 
Osterbrock, D. E., \& Shuder, J. M. 1982, ApJS, 49, 149

Peterson, B. M. 1993, PASP, 105, 247

Peterson, B. M., \& Wandel, A. 1999, ApJ, 521, L95

Peterson, B. M., Wanders, I., Bertram, R., Hunley, J. F., Pogge, R. W., \& Wagner, R. M. 1998, ApJ, 501, 82

Peterson, B. M., et al. 1991, ApJ, 368, 119 2000, ApJ, 542, 16

2002, ApJ, 581, 197

2004, ApJ, 613, 682 2005, ApJ, 632, 799

Tremaine, S., et al. 2002, ApJ, 574, 740
Ulrich, M.-H., \& Horne, K. 1996, MNRAS, 283, 748 van Groningen, E., \& Wanders, I. 1992, PASP, 104, 700

Vestergaard, M. 2002, ApJ, 571, 733 2004, ApJ, 601, 676

Vestergaard, M., \& Peterson, B. M. 2006, ApJ, 641, 689

Wandel, A., Peterson, B. M., \& Malkan, M. A. 1999, ApJ, 526, 579

White, R. J., \& Peterson, B. M. 1994, PASP, 106, 879

Yoshii, Y. 2002, in New Trends in Theoretical and Observational Cosmology, ed. K. Sato \& T. Shiromizu (Tokyo: Universal Academy), 235

Yoshii, Y., Kobayashi, Y., \& Minezaki, T. 2003, BAAS, 202, 3803 